\title{
Study on the Relationship Between Anxiety, Depression and Social Support of Caregivers of Mentally Retarded Children
}

\author{
Peng Qian ${ }^{\dagger}$, Li Wenxia ${ }^{\dagger}$, Ma Jun ${ }^{*}$, Wang Lijun, Liu Weiwei \\ School of Public Health and Management, Chongqing Medical University, Chongqing, China
}

Email address:

fairlily1117@163.com (Ma Jun)

${ }^{*}$ Corresponding author

$\uparrow$ Peng Qian and Li Wenxia are co-first authors.

\section{To cite this article:}

Peng Qian, Li Wenxia, Ma Jun, Wang Lijun, Liu Weiwei. Study on the Relationship Between Anxiety, Depression and Social Support of Caregivers of Mentally Retarded Children. Clinical Medicine Research. Vol. 9, No. 1, 2020, pp. 1-5. doi: 10.11648/j.cmr.20200901.11

Received: January 2, 2020; Accepted: January 18, 2020; Published: January 31, 2020

\begin{abstract}
Investigate the relationship between anxiety, depression and social support of caregivers of mentally retarded children, and provide further reference for their positive development. The Social Support Rating Scale and General Hospital Anxiety and Depression Scale were conducted among 111 caregivers of mentally retarded children in three special education institutions in a district of Chongqing by cluster sampling. SPSS22.0. was used to statistical analysis. The incidences of anxiety and depression in caregivers of mentally retarded children were $52.3 \%$ and $53.2 \%$ respectively. Caregivers of mentally retarded children in non-anxiety group and non-depression group scored significantly higher in subjective support, objective support, and total social support than those in obvious anxiety group and obvious depression group. Social support $(\gamma=-0.392, \gamma=-0.390)$ and subjective support $(\gamma=-0.335, \gamma=-0.325)$, objective support $(\gamma=-0.306, \gamma=-0.321)$ and support utilization $(\gamma=-0.235$, $\gamma=-0.224)$ for caregivers of mentally retarded children were negatively correlated with anxiety and depression. Multiple linear regression analysis showed that subjective support and objective support had negative effects on anxiety and depression. The anxiety, depression and social support of parents of mentally retarded children will interact each other. And providing diversified and service-oriented social support is conducive to alleviate their anxiety and depression, then promote their positive development.
\end{abstract}

Keywords: Mentally Retarded Children (MRC), Caregivers, Social Support, Anxiety, Depression

\section{Introduction}

Mentally retarded children (MRC) refers to children who have significant defects in intellectual function and adaptive behaviors that occurred before 18 years old [1]. The survey revealed that the prevalence of MRC is $1.2 \%$ in China [2] and MRC $(760,000)$ have the highest proportion among children with various disabilities (2.46 million) between the age of $6 \sim 14$ [3]. In the end of 2018, China has invested tens of billions in service facilities for children with disabilities including MRC [4], and most of MRC's survival and development rely on care and nursing from their caregivers due to their mental deficiencies. MRC's caregivers, as a connection of the output of services need for MRC and MRC's family with the supply of national administrative laws and regulations, are a visible and indispensable force to advocate rights of the disabled in China [5-6]. However, MRC's caregivers encountered significant psychological pressure, personal and family problems, economic burdens, and social pressures because of MRC [7-9], making them extremely prone to negative emotions. Such as anxiety and depression affect their own and children's favorable development and cause potential social consequences. Studies have shown that good external social support can help them alleviate individual stress, maintain a good emotional experience, and choose more effective coping styles [10-13]. Existing literature, related social policies and social services are only centered on MRC, while less attention is given to MRC's caregivers; in addition, most of the studies related to MRC's caregivers focus on their problems and dilemmas, few studies have specifically addressed relationship between their social support and 
negative emotions. Discussion above, this paper conducts an empirical study on the relationship between social support and anxiety, depression of MRC's caregivers, so as to provide a reference for promoting the positive development of MRC's caregivers.

\section{Materials and Methods}

\subsection{Research Objects}

Considering the particularity and sensitivity of MRC's caregivers, we selected subjects from three rehabilitation centers in the $\mathrm{S}$ district of Chongqing by cluster sampling, and eventually surveyed 111 subjects abiding by principle of voluntary participation. We released 152 questionnaires and collected 111 valid questionnaires. The effective recovery rate is $73.03 \%$. There were 17 fathers of MRC, 74 mothers of $\mathrm{MRC}$, and 20 grandparents of MRC respectively, and the average age was $(37.60 \pm 11.03)$ years old. When it comes to MRC's degree of intellectual disability, there were 27 milds, 41 moderates, 32 severes and 11 unclears.

\subsection{Research Methods}

We evaluated the level of subjects' anxiety and depression by General Hospital Anxiety and Depression Scale (GHAD) [14], which consists of two subscales: one to evaluate anxiety and another to evaluate depression. Each subscale is divided into no symptoms (negative 0-7), suspected symptoms (suspected positive 8-10), obvious symptoms (positive 11-21). The coefficients of Cronbach's $\alpha$ of anxiety subscale, depression subscale, and total scale are $0.81,0.73$, and 0.87 , respectively.

We evaluated subjects' social support through Social Support Rating Scale (SSR) formulated by Xiao Shuiyuan [15], which presents three-dimension structure, namely subjective support, objective support, and support utilization, and includes 10 items, which grade from 1 to 4 . The sum of 10 items is the total score of social support and the higher total score means the higher level of social support. The coefficients of Cronbach's $\alpha$ of SSR is 0.96 .

\subsection{Statistical Methods}

The data was statistically analyzed by SPSS 22.0 software, including ANOVA, correlation analysis, regression analysis, LSD test for pairwise comparison. $P<0.05$ considered statistically significant.

\section{Results}

\subsection{General Conditions of Anxiety, Depression and Social Support of MRC's Caregivers}

The anxiety average score of MRC's caregivers was (7.56 \pm 3.75 ) with 53 negatives, 29 suspected positives, 29 positives, and the incidence of anxiety was $52.3 \%$. While the depression average score of MRC's caregivers was (7.35 \pm 3.57 ) with 52 negatives, 41 suspected positives and 18 positives, and the incidence of depression was 53.2\%. The social support average score of MRC's caregivers was (35.50 \pm 7.35$)$, subjective support was $(21.29 \pm 4.38)$, objective support was (7.60 \pm 3.29$)$ and support utilization was $(6.60 \pm 1.74)$.

\subsection{Score Comparison of Anxiety, Depression and Social Support of MRC's Caregivers}

The subjects were divided into anxiety-free group, suspected anxiety group, obvious anxiety group on the basis of score range of anxiety subscale, and into depression-free group, suspected depression group, obvious depression group on the basis of score range of depression subscale to compare their difference on social support.

\subsubsection{Score Comparison of Anxiety and Social Support of MRC's Caregivers}

The results show that anxiety-free group and suspected anxiety group score are better than obvious anxiety group in social support, subjective support and objective support $(P<0.01)$; that anxiety-free group scores significantly higher than obvious anxiety group in support utilization $(P<0.05)$, while there is no significant difference in scores between the other group $(P>0.05)$. More details are shown in Table 1.

Table 1. Score Comparison of Anxiety and Social Support of MRC's Caregivers.

\begin{tabular}{|c|c|c|c|c|c|c|c|}
\hline & \multirow{2}{*}{$\begin{array}{l}\text { Anxiety-free group } \\
(\mathrm{N}=53) \text { (1) }\end{array}$} & \multirow{2}{*}{$\begin{array}{l}\text { Suspected anxiety } \\
\text { group }(N=29)(2)\end{array}$} & \multirow{2}{*}{$\begin{array}{l}\text { Obvious anxiety group } \\
(\mathrm{N}=29)(3)\end{array}$} & \multirow{2}{*}{$F$} & \multicolumn{3}{|c|}{$P$ (Pairwise comparison) } \\
\hline & & & & & (1)(2) & (1)(3) & (2)(3) \\
\hline Social support & $37.98 \pm 7.63$ & $35.55 \pm 6.13$ & $30.90 \pm 5.73$ & $10.160 *$ & 0.125 & $0.000 * *$ & $0.010 *$ \\
\hline Subjective support & $22.57 \pm 4.43$ & $21.28 \pm 3.87$ & $18.97 \pm 3.92$ & $7.018^{*}$ & 0.182 & $0.000 * *$ & $0.037 *$ \\
\hline Objective support & $8.45 \pm 3.50$ & $7.69 \pm 2.82$ & $5.97 \pm 2.80$ & $5.833^{*}$ & 0.298 & $0.001 * *$ & $0.040^{*}$ \\
\hline Support utilization & $6.69 \pm 1.78$ & $6.59 \pm 1.21$ & $5.97 \pm 1.97$ & $3.205^{*}$ & 0.342 & $0.013 *$ & 0.169 \\
\hline
\end{tabular}

Note: $* *: \mathrm{P}<0.01 ; *: \mathrm{P}<0.05$.

\subsubsection{Score Comparison of Depression and Social Support of MRC's Caregivers}

The research findings suggests that depression-free group scores significantly higher than suspected depression group and obvious depression group in social support and objective support $(P<0.01)$; that depression-free group scores significantly higher than obvious depression group in subjective support $(P<0.01)$; that suspected depression group scores significantly higher than obvious depression group in social support and subjective support $(P<0.05)$. While there is no significant difference in scores of support utilization among each group $(P>0.05)$. More details are shown in Table 2 . 
Table 2. Score Comparison of Depression and Social Support of MRC's Caregivers.

\begin{tabular}{|c|c|c|c|c|c|c|c|}
\hline & \multirow{2}{*}{$\begin{array}{l}\text { Depression-free } \\
\text { group }(\mathrm{N}=52)(1)\end{array}$} & \multirow{2}{*}{$\begin{array}{l}\text { Suspected depression } \\
\text { group }(\mathbf{N}=\mathbf{4 1})(2)\end{array}$} & \multirow{2}{*}{$\begin{array}{l}\text { Obvious depression } \\
\text { group }(\mathrm{N}=18) \text { (3) }\end{array}$} & \multirow{2}{*}{$\boldsymbol{F}$} & \multicolumn{3}{|c|}{$P$ (Pairwise comparison) } \\
\hline & & & & & (1)(2) & (1)(3) & (2)(3) \\
\hline Social support & $38.17 \pm 7.22$ & $34.37 \pm 6.48$ & $30.33 \pm 6.42$ & $9.700 *$ & $0.009 * *$ & $0.000 * *$ & $0.039 *$ \\
\hline Subjective support & $22.46 \pm 4.24$ & $21.12 \pm 3.89$ & $18.28 \pm 4.56$ & $6.786^{*}$ & 0.127 & $0.000 * *$ & $0.018^{*}$ \\
\hline Objective support & $8.73 \pm 3.21$ & $6.83 \pm 3.35$ & $6.11 \pm 2.24$ & $6.649 *$ & $0.004 * *$ & $0003 * *$ & 0.420 \\
\hline Support utilization & $6.98 \pm 1.67$ & $6.41 \pm 1.63$ & $5.94 \pm 1.98$ & 2.852 & - & - & - \\
\hline
\end{tabular}

Note: **: $P<0.01 ; *: P<0.05 ;-:$ no data.

\subsection{Correlation Analysis Between Anxiety, Depression and Social Support of MRC's Caregivers}

The results show that anxiety and depression of MRC's caregivers were significantly negatively correlated with social support, subjective support, and objective support $(P<0.01)$, and negatively correlated with support utilization $(P<0.05)$. More details are shown in Table 3.

Table 3. Correlation between Anxiety, Depression and Social Support of MRC's Caregivers ( $\gamma$ ).

\begin{tabular}{lllll}
\hline Index & Social support & Subjective support & Objective support & Support utilization \\
\hline Anxiety & $-0.392^{* *}$ & $-0.335^{* *}$ & $-0.306^{* *}$ & $-0.235^{*}$ \\
Depression & $-0.390^{* *}$ & $-0.325^{* *}$ & $-0.321^{* *}$ & $-0.224^{*}$ \\
\hline
\end{tabular}

Note: **: $P<0.01 ; *: P<0.05$.

\subsection{Regression Analysis of Factors Affecting Anxiety and Depression of MRC's Caregivers}

According to the correlation analysis above, anxiety score and depression score were regarded as the dependent variables and subjective support, objective support, support utilization, and social support as independent variables respectively when we performed multiple linear regression analysis with the inclusion criteria $\alpha=0.05$ and the excluding level $\beta=0.10$, and finally subjective support and objective support were enrolled in the regression equation. The results show that subjective support and objective support have a negative effect on anxiety and depression. More details are shown in Table 4.

Table 4. Multiple Regression Analysis of Factors Affecting Anxiety and Depression of MRC's Caregivers.

\begin{tabular}{llllll}
\hline Dependent variable & Independent variable & $\boldsymbol{B}$ & $\boldsymbol{S} \overline{\boldsymbol{x}}$ & $\boldsymbol{\beta}$ & $\boldsymbol{\boldsymbol { P }}$ \\
\hline \multirow{2}{*}{ Anxiety score } & Subjective support & -0.218 & 0.082 & 0.255 & -2.650 \\
& Objective support & -0.233 & 0.115 & -0.205 & -2.029 \\
\multirow{2}{*}{ Depression score } & Subjective support & -0.202 & 0.076 & -0.248 & -2.666 \\
& Objective support & -0.266 & 0.106 & -0.246 & -2.511 \\
\hline
\end{tabular}

\section{Discussion}

\subsection{High Incidence of Anxiety and Depression Among $M R C^{\prime}$ 's Caregivers}

The results of this study show that the prevalence of anxiety and depression in MRC's caregivers is $52.3 \%$ and $53.2 \%$, respectively, which indicates that MRC's caregivers are prone to anxiety and depression resembling the results of a survey of 119 MRC's caregivers by Bai Jinxin [16]. And the high incidence of anxiety/depression has a lot to do with MRC. The possible reasons are as follows: (1) The majority of MRC's caregivers are unable to fully embrace the reality, have psychological stress like doubt, anxiety, depression and even affect their family kinship and family functions on account of the specificity and the extremely low cure rate of mental retardation. (2) Due to the huge economic burden and slow progress of rehabilitation training, MRC's caregivers often fall into anxiety, depression and other bad emotions, which not only affects their own mental health, but may also lead to adverse social consequences. (3) MRC's caregivers now confront with "soft pressures" in culture and systems and the difficulties of MRC's future in a society where the value and existence of MRC are not fully understood, which creates heavy psychological burden. These reasons above indicate that we need to provide the necessary psychological support and social assistance for MRC's caregivers, to help them reasonably regulate their emotions, relieve their stress, and actively respond to social life considering their fragility and specialty.

\subsection{Close Relationship Between Anxiety, Depression and Social Support of MRC's Caregivers}

The results of this study show that social support scores of MRC's caregivers vary with different levels of anxiety and depression, suggested that anxiety and depression of MRC's caregivers will affect their access to social support. One possible reason is that MRC's caregivers lower their enthusiasm and initiative to seek social support for they are in negative emotions like anxiety and depression, so there is differences in social support. Social support utilization of MRC's is lower than the national norm (6.92) [15], and there are only differences in social support utilization of MRC between anxiety-free group and the obvious anxiety group, 
which may relate with the "emotional disturbance" in the use of social support. For example, although some MRC's caregivers can get support from others, they may refuse it because serious stigmatization of MRC impairs their self-esteem; Interpersonal support is an interactive process, some of them never ask for help or gently refuse to make use of social support because they are unable to give back due to their predicament. Therefore, it is necessary to conduct psychological counseling and intervention for MRC's caregivers, so that they can understand and utilize the social support properly. It is also found that social support of MRC's caregivers gradually decreases with the worsening of depression while social support of MRC's caregivers declines when the anxiety reaches a certain level, which is likely to involve the different severity of anxiety and depression -- for depression may be more pathological. Moreover, the results of the correlation analysis show that social support and its dimensions of MRC's caregivers are negatively related to their anxiety and depression, indicating that social support can alleviate their anxiety and depression, which is similar to the findings of Wu Yi [17]. Tian Boqiong and Shen Renhong's research also found that social support is generally positively related to the positive contributions of MRC's families [18] so that we should provide them with more care, support, and available social support to promote their positive development when we confront MRC's caregivers under tremendous psychological pressure.

\subsection{Effects of Subjective Support, Objective Support on Anxiety and Depression of MRC's Caregivers}

The results of regression analysis show that subjective support and objective support have a negative effect on the anxiety and depression of MRC's caregivers, that is, MRC's caregivers with a high degree of subjective and/or objective support are less likely to occur anxiety and depression. Not only that, research has also found that the level of life quality of MRC's caregivers is significantly positively related to the level of social support [19]. Here are the possible reasons: Firstly, their subjective emotional experiences: being respected, being understood, and being supported, although not the tangible support, which means being accepted and understood in social life, and the perceived reality is their psychological reality, which is beneficial to maintain a good emotional experience and affect their behavior and development [20]. Li Fangfang and Yang Liu's research has also found that there is a significant negative correlation between care burnout and social support for MRC's caregivers [21]. Secondly, visible and targeted objective support of MRC's caregivers, which encountered care pressure, economic pressure and social pressure will effectively reduce their burden and facilitate their active response in daily life. Other studies have also pointed out that it can gradually form a multi-subject social support system and alleviate their care pressure by means of constructing their social support network [22].

\section{Conclusion}

Taken together, the anxiety, depression and social support of MRC's caregivers will interact each other. In order to facilitate the positive development of MRC's caregivers, we need to provide them with instrumental support to effectively reduce their burden, and psychological and emotional support. The whole society must correctly recognize and accept MRC and MRC's caregivers, so that they can form a correct understanding of values and increase the utilization of social support. That is to provide diversified and service-oriented social support for MRC's caregivers and to drive them to form a more comprehensive and objective response to social life and to develop towards a benign direction, so as to benefit the health and harmony of the whole society.

\section{References}

[1] AAIDD. "Definition of intellectual disability" [EB/OL]. http://www.aaidd.org/content100.cfmnavID=21, March 2011.

[2] National collaboration group, Zu QH, Lei FW, Zhang ZX, et al. "National epidemiological survey of mental retardation among children aged 0-14 years old-prevalence of mental retardation"[J]. Chinese Journal of Reproductive Health, 1991 (3): $105-110$.

[3] National Bureau of statistics and leading group of the second national sampling survey of disabled persons: Bulletin of main data of the second national sampling survey of disabled persons $\begin{array}{llll}\text { in } 2006 & \text { (No. } & 2 \text { ) } \\ \text { [EB/OL] }\end{array}$ http://www.cdpf.org.cn/sjzx/cjrgk/200711/t20071121_387540 .shtml, 2007-05-28.

[4] China Disabled Persons' Federation. 2018 statistical bulletin of disabled persons' career development [disabled persons' $\begin{array}{llll}\text { Federation } & \text { (2019) No. } & 18] \\ \text { [EB/OL]. }\end{array}$ http://www.cdpf.org.cn/zcwj/zxwj/201903/t20190327_.shtml, 2019-03-27.

[5] Liao Y. "Research on the scope of state, society and citizen's responsibility for the protection of the rights and interests of the disabled" [J]. Journal of Jianghan University (Social Science Edition), 2013, 30 (5): 50-56.

[6] Li XH, Zhang FQ. "Rights Protection for Persons with Intellectual Disability and Mental Disorder: A Family Perspective" [J]. Journal of Southwest University of Political Science and Law, 2018, 20 (5): 58-65.

[7] Peng H, Zhou HY, Cheng SY, et al. "A survey of parents' psychological stress of preschool disabled children in Beijing" [J]. Chinese Journal of Special Education, 2010 (5): 12-17.

[8] Chan, K. K. S. \&Lam, C. B. "Self-stigma among parents of children with autism spectrum disorder" $[\mathrm{J}]$. Research in Autism Spectrum Disorders, 2018, 48, 44-52.

[9] Tang, L. \& Bie, B. "The stigma of autism in china: an analysis of newspaper portrayals of autism between 2003 and 2012"[J]. Health Communication, 2016, 31 (4), 445-452.

[10] Gong YX. "A survey of the relationship between social support and health" $[\mathrm{J}]$. Journal of Developments in Psychology, 1994, 2 (2): 34-39. 
[11] Li XH. "The influence of autistic children on family and family coping strategies: an understanding framework based on literature review'[J]. Social Work and Management, 2018, 18 (4): $34-39$.

[12] Lin, L. Y. "Coping strategies, care giving burden, and depressive symptoms of Taiwanese mothers of adolescents with autism spectrum disorder" $[J]$. Research in Autism Spectrum Disorders, 2015, 15, 1-9.

[13] Pederson, S. Revenson, T. "Parental illness, family functioning, and adolescent well-being: A family ecology framework to guide research"[J]. Journal of Family Psychology, 2005, 19 (3): 404-409.

[14] ZIGMOND A S, SNAITH R P. "The hospital anxiety and depression scale" $[J]$. Acta Psychiatr Scand, 1983, 67 (6): 361-370.

[15] Wang XD, Wang XL, Ma H. "Handbook of mental health assessment scale" [J]. Chinese Mental Health Journal, 1999 (Supplement): 31-35.

[16] Bai JX, Sun L, Guan SS, et al. "Study on the relationship between anxiety, depression and coping style of parents of mentally retarded children" [J]. Nursing research, 2015, 29 (31): 3899-3901.
[17] Wu M. "The Relationship of Parents' Social Support and Anxiety from Autism Children" [J]. Journal of Gannan Normal University, 2010, 31 (2): 100-102.

[18] Tian BQ, Zeng SL, Lu XL, Shen RH. "Positive Contribution of Families Having Preschool Children with Intellectual Disabilities and Its Relationship with Social Support" [J]. XUEQIAN JIAOYU YANJIU, 2018, 2: 35-51.

[19] Li JY. "Corelationship research on the quality of life and social support among parents of children with intellectual and development disabilitiest" [D]. Wuhan: Central China Normal University, 2015.

[20] Thoits PA. "Dimensions of life events that influence psychological distress: an evaluation and synthesis of the literature". In: Kaplan H. et (eds): Psychological Stress lasted. New York, Academic Press, 1983: 33-103.

[21] Li FF. "Relationship research on Care burnout, Caregiver burden and Social support among Family Caregivers of Special Children" [D]. Chongqing: Southwest University, 2016.

[22] Hao WW. "Construction of social support network for the mentally disabled children female family caregivers - Base on the practical study of social support theory" [D]. Guangzhou: South China University of Technology, 2016. 